\title{
Strongly correlated crystal-field approach to Mott insulator $\mathrm{LaCoO}_{3}$
}

\author{
R.J. Radwansk:* \\ Center for Solid State Physics, S ${ }^{\text {nt }}$ Filip 5, 31-150 Krakow, Poland, \\ Institute of Physics, Pedagogical University, 30-084 Krakow, Poland \\ Z. Ropka \\ Center for Solid State Physics, ${ }^{\text {nt }}$ Filip 5, 31-150 Krakow, Poland
}

\begin{abstract}
Our success in description of recent electron-spin-resonance results on Mott insulator $\mathrm{LaCoO}_{3}$, Phys. Rev. B 67 (2003) 172401, lies in taking into account strong electron correlations among $d$ electrons and the relativistic spin-orbit coupling. In the developed by us Quantum Atomistic Solid State Theory (QUASST) we assume that the atomic-like integrity of the $3 \mathrm{~d}^{6}$ system is preserved in the $\mathrm{Co}^{3+}$ ion in $\mathrm{LaCoO}_{3}$ and that intra-atomic correlations are much stronger than crystal field interactions. We conclude that in $\mathrm{LaCoO}_{3}$ there is no intermediate spin state as came out from band-structure calculations. The excited states originate from the high-spin ${ }^{5} T_{2 g}$ term, being $12 \mathrm{meV}$ above the ground ${ }^{1} A_{1}$ state. We are convinced that many-electron CEF approach with strong correlations and the atomic-scale orbital magnetism is physically adequate approach to $3 d$ oxides.
\end{abstract}

Keywords: Mott insulator, crystal field, spin-orbit coupling, $\mathrm{LaCoO}_{3}$

$\mathrm{LaCoO}_{3}$ attracts much attention in recent 50 years due to its insulating non-magnetic ground state and the significant violation of the Curie-Weiss law at low temperatures in the temperature dependence of the paramagnetic susceptibility $\chi(T)$ exhibiting a pronounced maximum at $100 \mathrm{~K}$ 1]. This $\chi(T)$ dependence is customarily discussed in terms of successive changes of spin states with the increasing temperature as low-spin (LS), intermediate-spin (IS) and high-spin (HS) states $[2,[3,4,[5]$.

Recent experimental observation of well-defined localized states by Noguchi et al. [6] by means of Electron Spin Resonance (ESR) measurements sharply contradict, according to us, band-structure calculations [2, 3, 4, 5], that yield the continuous energy spectrum for $3 d$ states spread over 6 eV. The developed by us Quantum Atomistic Solid State Theory 7], 8], incorporating the manyelectron crystal-field theory was the only present theory that was prepared for the ESR results revealing discrete energy states. We have provided [9] a complete explanation of Noguchi et al.'s results making use of our earlier long lasting studies on the CEF-field effect in combination with

*URL: http://www.css-physics.edu.pl Electronic address: sfradwan@cyf-kr.edu.pl 
the spin-orbit coupling on properties of $3 d$ oxides and of $\mathrm{LaCoO}_{3}[10,11,12]$.

In the ESR experiment Noguchi et al. [6] have derived $g$ tensor, $g_{\|}=3.35, g_{\perp}=3.55$, of the excited quasi-triplet described by an effective spin $\tilde{S}=1$ with a splitting $D=+4.90 \mathrm{~cm}^{-1}(=7.056$ $\mathrm{K}=0.6 \mathrm{meV}$ ) with external magnetic field applied along different crystallographic directions of single-crystalline $\mathrm{LaCoO}_{3}$. We have proven that these localized states originate from the ${ }^{5} \mathrm{D}$ term and that the $g$ tensor and the behavior of excitation energies in fields up to $60 \mathrm{~T}$ is very well described by the crystal-field Hamiltonian with taking into account the spin-orbit interactions [9].

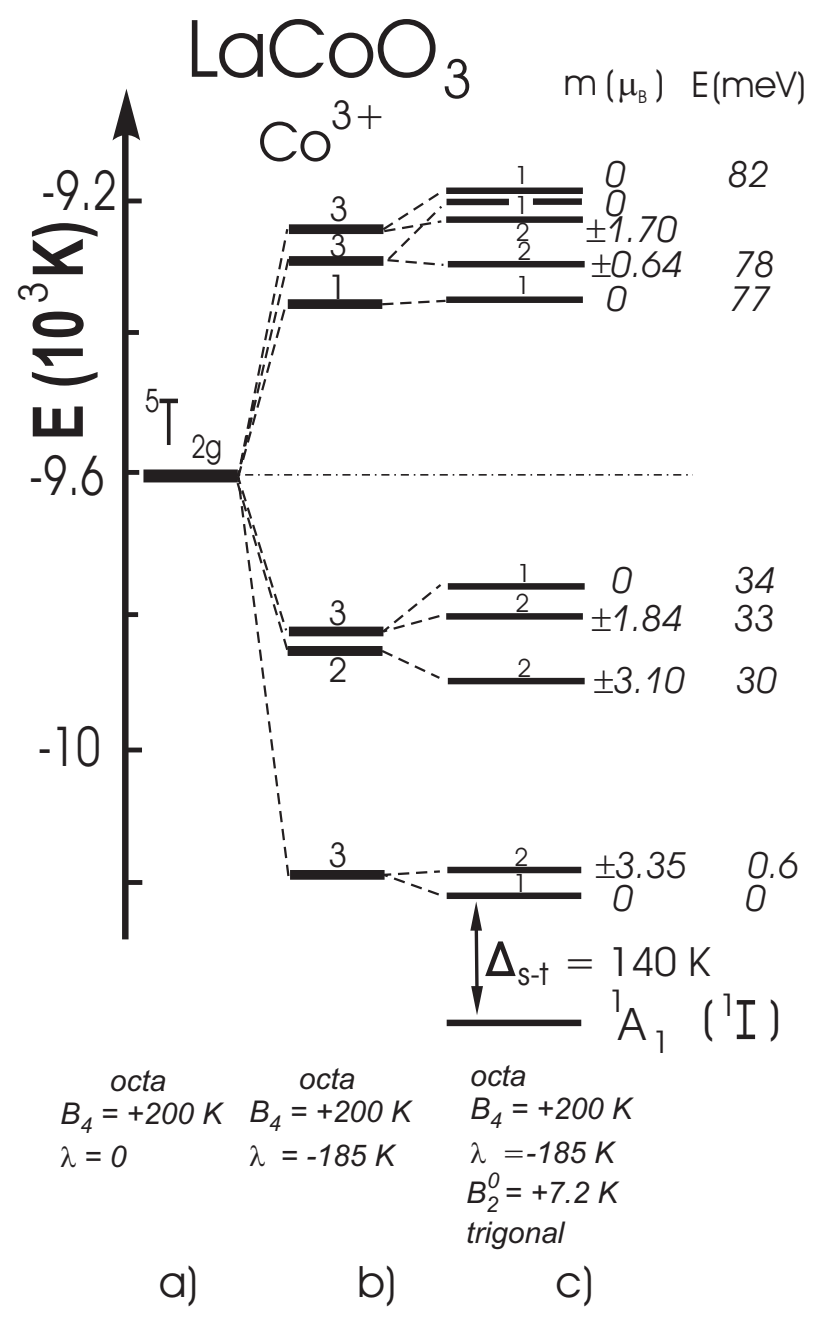

FIG. 1: Calculated low-energy localized states of the $\mathrm{Co}^{3+}$ ion in $\mathrm{LaCoO}_{3}$ originating from the ${ }^{5} T_{2 g}$ subterm with the ground state ${ }^{1} A_{1}$.

The aim of this paper is to discuss the role of electron correlations and their manifestation in $\mathrm{LaCoO}_{3}$ as well as the physics of low-spin (LS), intermediate-spin (IS) and high-spin (HS) states. We have proven that the excited triplet state originates from the atomic-like high-spin $(S=2)$ term 


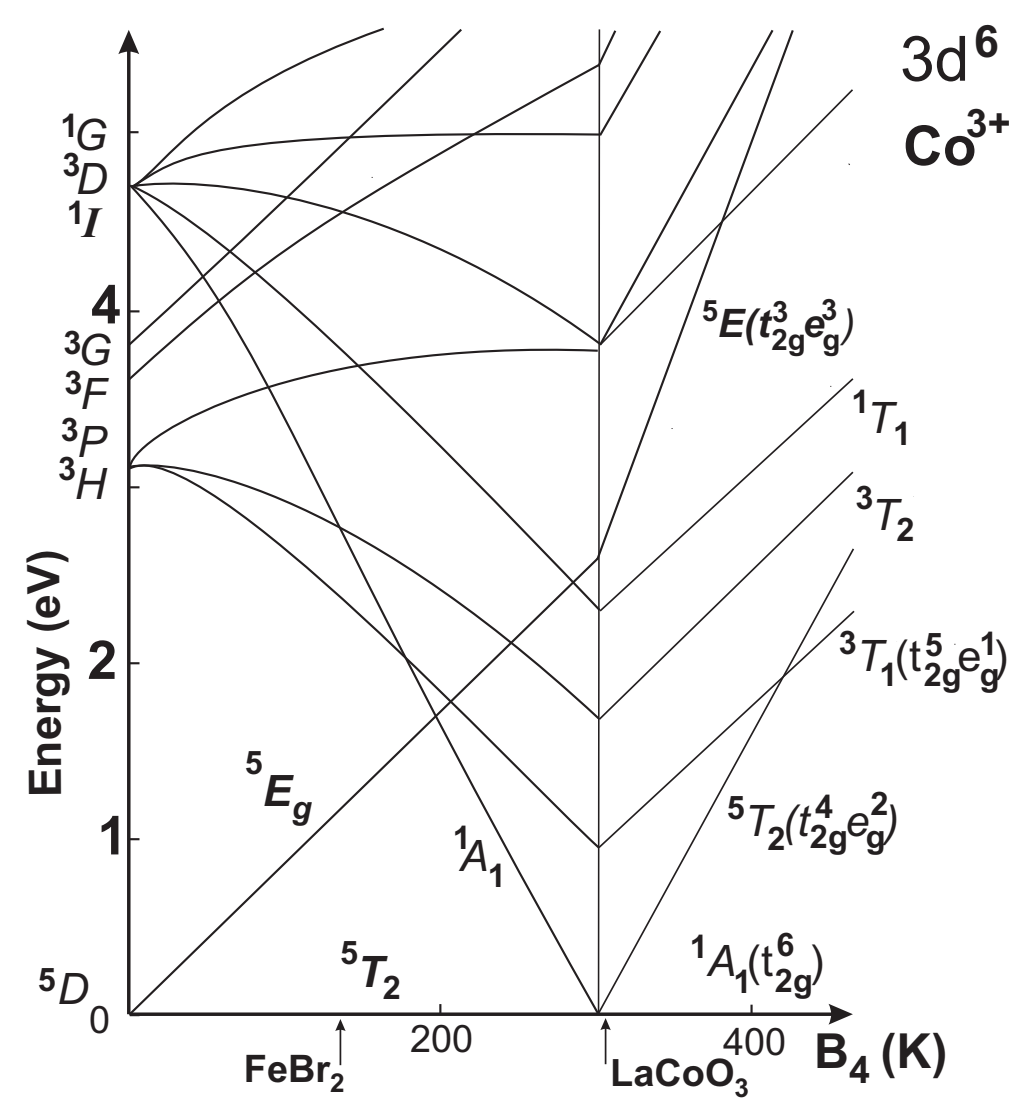

FIG. 2: Influence of the strength of the octahedral crystal field on the atomic state of the $\mathrm{Co}^{3+}$ ion $\left(3 \mathrm{~d}^{6}\right.$ system) (Tanabe-Sugano diagram) 13]. Data for $\mathrm{FeBr}_{2}$ are after Ref. 14].

${ }^{5} \mathrm{D}$ of the $3 d^{6}$ electron system existing in the $\mathrm{Co}^{3+}$ ion because i) such the triplet is expected for the octahedral crystal field in the presence of the spin-orbit interactions 10], ii) by perfect reproduction of the $g$ tensor and iii) the splitting of the triplet by the rhombohedral distortion as well as iv) the reproduction of the behavior of excitation energies in fields $\mathbf{B}$ up to $60 \mathrm{~T}$ for different crystallographic directions with the single-ion Hamiltonian for the term ${ }^{5} D$ with $S=2$ and $L=2$ :

$$
H_{d}=H_{c u b}\left(L, L_{z}\right)+\lambda_{s-o} L \cdot S+B_{2}^{0} O_{2}^{0}\left(L, L_{z}\right)+\mu_{B}\left(L+g_{s} S\right) \cdot \mathbf{B}
$$

The good description proves the energy scales assumed in QUASST for consideration of $3 d$ oxides: the dominant energy is the octahedral CEF interactions $(2-3 \mathrm{eV})$, weaker the spin-orbit coupling $(16 \mathrm{meV})$ and the weakest are off-octahedral distortions $(1 \mathrm{meV})$.

In the shown description we use only 3 parameters: $\mathrm{B}_{4}$ (from $a b$ initio calculations for the $d^{6}$ configuration $\mathrm{B}_{4}$ turns out to be positive for the $\mathrm{O}^{2-}$ octahedron with the found value of 280-300 $\mathrm{K}), \lambda_{s-o}$ and $\mathrm{B}_{2}^{0}$. All of them have clear physical meaning.

Detailed electronic structure of $\mathrm{LaCoO}_{3}$ is shown in Fig. 1. At lowest temperatures the only 


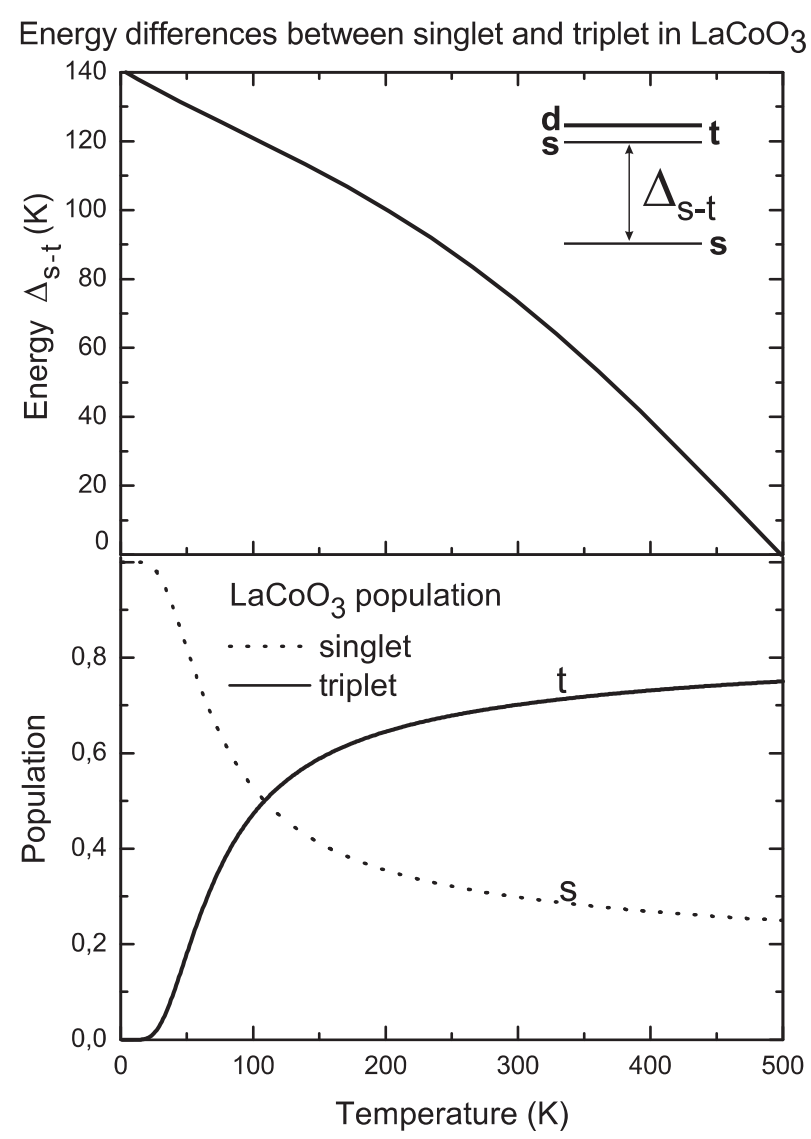

FIG. 3: Temperature dependence of the energy separation $\Delta_{s-t}$ between the lowest singlet and the excited triplet. This dependence is inferred from the thermal expansion and experimental evaluation of temperature dependence of the Co-O distance. In the bottom part the calculated results for the population of the singlet and triplet states is shown. The population of the triplet state can be understood as population of the $\mathrm{Co}^{3+}$ ions in the high-spin state.

populated state is ${ }^{1} A_{1}$ subterm that is a singlet. It is the reason for the diamagnetism of $\mathrm{LaCoO}_{3}$ as all atoms are in singlet ground states. The electronic structure below $25 \mathrm{meV}$ determines magnetic and electronic properties below room temperatures.

We conclude that:

i) $\mathrm{LaCoO}_{3}$ is the Mott insulator. It is insulator, despite of the incomplete $3 d$ shell, due to strong atomic-like electron correlations, that in QUASST we recognize as predominantly intra-atomic.

ii) There is no intermediate spin state in contrast to band-structure calculations of Ref. 2 and numerous further quotations.

iii) The low spin state is the singlet ${ }^{1} A_{1}$ subterm - it originates from the atomic term ${ }^{1} I$ and becomes the ground state by octahedral crystal-field interactions due to the large orbital moment $(L=6)$ of the ${ }^{1} I$ term (Fig. 2). 
iv) Detailed description of magnetic and electronic properties of $\mathrm{LaCoO}_{3}$ is hampered as the electronic structure shown in Fig. 1 changes with temperature. Due to the thermal expansion the energy separation $\Delta_{s-t}$ decreases becoming zero at, according to our estimations, about $500 \mathrm{~K}$, Fig. 3. The applied pressure causes an increase of $\Delta_{s-t}$ what will manifest in the shift of the $\chi(T)$ maximum to higher temperatures. With increasing temperature the population of the high-spin state rapidly grows - already at $100 \mathrm{~K}$ the LS- and HS-state population becomes equal. Above 100 $\mathrm{K}$ the HS state is more populated than LS state.

v) So fast population of the HS state enables the development of the orbital ordering with temperature as the Co ions in the HS state have non-spherical shape of their charge cloud.

vi) We think that the Co sublattice can lose homogenity and $2 / 3$ of Co ions experience stronger the octahedral crystal field and have larger value of $\Delta_{s-t}$ than, say $150 \mathrm{meV}$. Due to this larger $\Delta_{s-t}$ they only slightly contribute to the macroscopically-observed susceptibility. But this point needs further studies.

[1] V.G. Bhide, D.S. Rajoria, G. Rama Rao, and C.N.R. Rao, Phys. Rev. B 6 (1972) 1021.

[2] M.A. Korotin, S.Yu. Ezhov, I.V. Solovyev, V.I. Anisimov, D.I. Khomskii, and G.A. Sawatzky, Phys. Rev. B 54 (1996) 5309.

[3] N. Solovyev, N. Hamada, and K. Terakura, Phys. Rev. B 53 (1996) 7158.

[4] P. Ravindran, P.A. Korzhavyi, H. Fjellvag, and A. Kjekshus, Phys. Rev. B 60 (1999) 16423.

[5] P.G. Radaelli and S.-W.Cheong, Phys. Rev. B 66 (2002) 094408.

[6] S. Noguchi, S. Kawamata, K. Okuda, H. Nojiri, and M. Motokawa, Phys. Rev. B 66 (2002) 094404.

[7] R.J. Radwanski and Z. Ropka, Quantum Atomistic Solid-State Theory; cond-mat/0010081.

[8] R.J. Radwanski, R. Michalski, and Z. Ropka, Acta Physica Pol. B 31 (2000) 3079.

[9] Z. Ropka, R. Michalski, and R.J. Radwanski, Phys. Rev. B 67 (2003) 172401.

[10] R.J. Radwanski and Z. Ropka, Relativistic effects in the electronic structure for the 3d paramagnetic ion, Report of CSSP-4/97; cond-mat/9907140

[11] Z. Ropka and R.J. Radwanski, Physica B 312\&313 (2002) 777.

[12] Z. Ropka and R.J. Radwanski, The $3 d$ electron states in $\mathrm{LaCoO}_{3}$; cond-mat/0012228.

[13] This Tanabe-Sugano diagram has been checked for us by R. Herchel and R. Boca from University of Bratyslava (Slovakia) for $\mathrm{Dq}=3666 \mathrm{~K}$. The authors are grateful to them.

[14] Z. Ropka, R. Michalski, and R.J. Radwanski, Phys. Rev. B 63 (2001) 172404. 